\title{
Global Vaccine and Immunology - Open Access
}

\author{
Charles J. Malemud* \\ Case Western Reserve University, USA
}

It is my distinct honor and privilege to have accepted an invitation to become Founding Editor and Editor-in-Chief (EIC) of Global Vaccine and Immunology (GVI), an online peer-reviewed open access journal. In my capacity as EIC and on behalf of our Editorial Board members we will be dedicated to providing the scientific community with the results of important advances in vaccine development as well as recognizing that only through basic and fundamental research focusing on immunological mechanisms, rigorous pre-clinical evaluation and by carefully-designed clinical trials will novel vaccine formulations designed to thwart world-wide infectious diseases and other human disease conditions be developed.

It is certain that vaccines have positively affected the health and well-being of persons throughout the world. A perfect example of how scientists in conjunction with the biopharmaceutical industry can work together was the response of these groups to the recent outbreak of Ebola virus disease (EVD) infection in the West African nations, Liberia, Guinea and Sierra Leone. Since most conventional therapies do not result in a cure of EVD, positive steps needed to be were taken to rapidly develop and test a novel vaccine formulation for treatingEVD. In fact, vaccine candidates with promising results in nonhuman primates were shown to have protective effects against lethal EVD infection. However at this juncture, and as a starting point, Phase I clinical trials will be employed to test the effect of administering the vaccine to healthy human subjects as well as the extent to which this vaccine results in an immune response. This Phase I clinical trial will also identify any untoward adverse and significant side-effects of the vaccine as well as determining the appropriate dosage to be employed in EVD-infected subjects. Thus, as EIC of GVI I encourage those basic research scientists and public health personnel to submit manuscripts describing this overall experience with EVD disease. It is not often in our lifetime that we are faced with such a health-related calamity in combination with such a strong potential for a world-wide pandemic, so recent advances in EVD vaccine development serves as a perfect example of how the scientific community meets such a challenge.

GVI will also be dedicated to publishing novel findings relevant to fundamental immunology in both the human and veterinary

Copyright: (C2015 Malemud CJ. This is an open-access article distributed under the terms of the Creative Commons Attribution License, which permits unrestricted use, distribution, and reproduction in any medium, provided the original author and source are credited. sciences. We encourage scientists world-wide to consider submitting manuscripts in all topics of immunology, including cellular and molecular immunology, microbial and fungal immunology, allergy, transplantation immunology, cancer immunology, as well as research studies relevant to the immunodeficiency diseases andin the pharmacology that serves as the underpinning for developing drugs to treat human immunologically-based diseases. One such area that I will be particularly interested in receiving manuscripts would be in fields that are not generally considered to be a focus of vaccine-research. For example, one such area could include the potential for vaccine development for the prophylactic therapy of autoimmune diseases, such as rheumatoid arthritis (RA). Indeed current RA research provided compelling evidence that anti-CCP antibodies were more specific than rheumatoid factor for making the diagnosis of rheumatoid arthritis. Furthermore, the presence of anti-CCP antibodies may be a better predictor of erosive synovial joint disease. Thus, vaccine development for use in such individuals prior to the initial signs and symptoms of clinical RA would constitute a major advance in the long-term goal of researchers to eventually "cure" RA.

We also look forward to receiving manuscripts that would address some of the more controversial aspects associated with vaccine development. In that regard, we must consider aspects of social conscience that could preclude, for example, the use of human fetal tissue for use in vaccine development despite the fact that these tissue culture systems have been successfully employed for the development of polio, chickenpox and rubella vaccines.

Finally, The Editorial Board of GVI is committed to the rigorous peer-review of all submitted manuscripts, including original research, observational studies, hypothesis and theory, methods and techniques as well as reports on advancing technologies. We also propose to initiate "Special Thematic Issues" which would take the form of invited mini-reviews which would be vetted by "guest" editors .

In closing GVI looks forward to serving as a publishing venue for the scientific community through our commitment to maintaining the integrity of the scientific method. We trust that you will consider submitting your research findings to GVI in the near future.

Correspondence to: Charles J. Malemud, Professor Medicine and Anatomy, Case Western Reserve University, USA, Tel: 0012165361945 ; E-mail: cjm4@case.edu

Received: December 07, 2015; Accepted: December 08, 2015; Published: December 10, 2015 